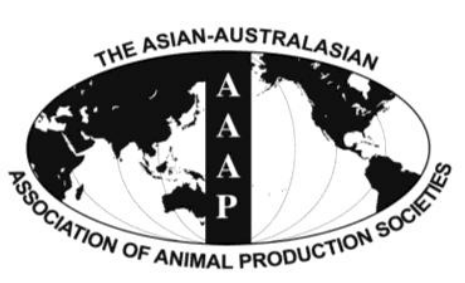

Open Access

Asian Australas. J. Anim. Sci.

Vol. 28, No. 1 : 86-94 January 2015

http://dx.doi.org/10.5713/ajas.14.0109

www.ajas.info

pISSN $1011-2367$ elSSN 1976-5517

\title{
Determination and Prediction of the Amino Acid Digestibility of Sunflower Seed Meals in Growing Pigs
}

\author{
J. D. Liu, Q. Y. Li, Z. K. Zeng, P. Li, X. Xu, H. L. Wang, S. Zhang, and X. S. Piao* \\ Ministry of Agriculture Feed Industry Centre, State Key Laboratory of Animal Nutrition, \\ China Agricultural University, Beijing 100193, China
}

\begin{abstract}
This experiment was conducted to evaluate the chemical composition and amino acid (AA) digestibility of sunflower seed meal (SFSM) and to use this data to develop prediction equations for estimating AA digestibility for growing pigs. Ten SFSM were collected from five provinces in China. Twelve barrows $(38.8 \pm 4.6 \mathrm{~kg}$ ), fitted with ileal T-cannula were allotted into two $6 \times 6 \mathrm{Latin}$ square designs. Each of six experimental periods comprised a 5-d adaption period followed by a 2-d collection of ileal digesta. The ten test diets contained 50\% SFSM as the sole source of AA. Another nitrogen-free diet was used to measure the basal endogenous losses of crude protein $(\mathrm{CP})$ and AA. Chromic oxide $(0.3 \%)$ was used as an inert marker in each diet. There was considerable variation $(\mathrm{CV}>10 \%)$ among the ten SFSM in chemical composition (dry matter [DM]). The concentration of CP and ether extract (EE) ranged from $29.33 \%$ to $39.09 \%$ and $0.88 \%$ to $11.33 \%$, respectively. Crude fibre (CF), neutral detergent fibre and acid detergent fibre ranged from $21.46 \%$ to $36.42 \%, 38.15 \%$ to $55.40 \%$, and $24.59 \%$ to $37.34 \%$, respectively. There was variation among the ten SFSM in apparent ileal digestibility (AID) and standardized ileal digestibility (SID) for lysine and threonine, which ranged from 63.16 to 79.21 and $55.19 \%$ to $72.04 \%$ for AID and $67.03 \%$ to $82.07 \%$ and $61.97 \%$ to $77.01 \%$ for SID, respectively. The variation in CP and methionine ranged from $60.13 \%$ to $74.72 \%$ and $74.79 \%$ to $88.60 \%$ for AID and $66.70 \%$ to $79.31 \%$ and $77.16 \%$ to $90.27 \%$ for SID, respectively. Methionine was a good indicator to predict AA digestibility. These results indicate that conventional chemical composition of SFSM was variable $(\mathrm{CV}>10 \%)$ among the ten SFSM (DM). The results of AID, SID and prediction equations could be used to evaluate the digestibility of SFSM in growing pigs. (Key Words: Sunflower Seed Meal, Chemical Composition, Amino Acid Digestibility, Growing Pigs, Prediction Equations)
\end{abstract}

\section{INTRODUCTION}

Sunflower (Helianthus annuus, Asteraceae) is one of the most widely cultivated oil crops in the world (Flagella et al., 2002). The world-wild production of sunflower seed reached 37.08 million tonnes and subsequently produced 15.22 million tonne of oil (FAO, 2012). Together with soybeans, cottonseeds and canola (rapeseed), sunflower seeds are one of the major oilseeds produced in the world (Salunkhe et al., 1992).

Oilseed by-products play an important role in supplying plant protein (Church and Kellems, 1998). Soybean meal

\footnotetext{
* Corresponding Author: X. S. Piao. Tel: +86-10-62733588, Fax: +86-10-62733688, E-mail: piaoxsh@mafic.ac.cn

Submitted Feb. 13, 2014; Revised Apr. 25, 2014; Accepted Jun. 23, 2014
}

(SBM) is often referred to as the gold standard compared with other protein sources (Cromwell, 2000; Zhang et al., 2013). The demand for soybean is increasing due to its inclusion in livestock feed and also its use for human consumption (Sasipriya and Siddhuraju, 2013). In order to satisfy the need for protein resources, other materials such as rapeseed meal (Baidoo et al., 1987; Brand et al., 2001; Opalkal et al., 2001; Zhang et al., 2012), cottonseed meal (Noland et al., 1968; Tanksley et al., 1981) and peanut meal (Batal et al., 2005; Sulabo et al., 2013) have been used to replace SBM in the feed industry.

Sunflower seed meal (SFSM) is a by-product of the oil extraction of sunflowers and could be an important protein resource for use in animal diets. Solvent extracted sunflower seed meal has an average concentration of crude protein $(\mathrm{CP})$ of $30.7 \%$ and a higher concentration of 
Methionine (Met) than solvent extracted SBM, but has less lysine (Lys) than SBM (NRC, 2012). Another characteristic of SFSM is that it is not known to have antinutritional factors such as those found in soyabean, cottonseed and rapeseed meals. Although sunflower seed contains $1.56 \%$ chlorogenic acid (Milic et al., 1968), its concentration in SFSM does not lead to toxicity effects (Senkoylu and Dale, 1999). Sunflower seed meal diets supplemented with Lys can be used as a replacement for SBM in growing pigs (Seerley et al., 1974). However, the digestibility of these nutrients may vary considerably resulting in rather inaccurate measures for the nutritional value of actual batches of feed (Just et al., 1983). Therefore, the objective of this study was to compare the chemical composition of different SFSM and determine the apparent ileal digestibility (AID) and standardized ileal digestibility (SID) of CP and amino acid (AA) of SFSM fed to growing pigs. In addition, the results of the chemical analysis were used to establish prediction equations for AID and SID that could be applied in future commercial practice.

\section{MATERIALS AND METHODS}

This experiment was conducted at the China Agricultural University (Beijing, China). The Institutional Animal Care and Use Commitee at China Agricultural University approved the protocol for the experiment. All the pigs had been used in another experiment before being placed in this experiment.

\section{Animals and housing}

The experiment was conducted in the Metabolism Laboratory of the Ministry of Agriculture Feed Industry Centre, Beijing. Temperature and humidity of the room were automatically controlled at $18^{\circ} \mathrm{C}$ to $21^{\circ} \mathrm{C}$ and $50 \%$ to $65 \%$.

Twelve barrows (Duroc $\times$ Landrace $\times$ Yorkshire) with an initial body body weight (BW) $38.8 \pm 4.6 \mathrm{~kg}$ were fitted with a simple T-cannula in the distal ileum (Stein et al., 1998). The barrows were individually placed in stainless-steel metabolism crates $\left(1.4 \times 0.45 \times 0.6 \mathrm{~m}^{3}\right)$. Each crate was installed with a one-hole feeder and a nipple drinker.

\section{Source of sunflower seed meal and experiment design}

Ten sunflower seed meals were collected from five provinces in China which are representative of more than $80 \%$ of the output of China. The chemical composition of ten SFSM differed from each other (Tables 1 and 2).

The 12 barrows were allocated into two $6 \times 6$ Latin square designs according to their initial BW with 6 pigs for each Latin square. Each Latin square contained 5 SFSM which were the only source of AA and one nitrogen-free diet which was used to estimate basal ileal endogenous losses of CP and AA. The diets used in this experiment were prepared based on the chemical composition of the feed ingredients (Tables 3 and 4). All diets were fed in mash form.

\section{Sample collection}

The daily feed intake at the beginning of each period was set at 4\% of BW (Adeola, 2001). During each of the 6 experimental periods, the first $5 \mathrm{~d}$ were for adaptation to the diet. On d 6 and 7, ileal digesta samples were collected from 8:00 to 16:00 h. Cannulas were opened and plastic bags were fastened with the help of a rubber band in order to collect the digesta flowing into the bags. Every 30 minutes, the plastic bags were replaced and digesta samples were taken and stored at $-20^{\circ} \mathrm{C}$. Ileal digesta samples were thawed and mixed at the end of every two day collection period. All the samples were mixed and lyophilised in a Vacuum-Freeze Dryer (Tofflon Freezing Drying Systems, Minhang District, Shanghai, China) and passed a $1 \mathrm{~mm}$ screen before a sub-sample was obtained for chemical analysis.

Table 1. Analyzed chemical composition of sunflower seed meal (\% DM)

\begin{tabular}{|c|c|c|c|c|c|c|c|c|c|c|c|c|c|c|}
\hline \multirow{2}{*}{ Item } & \multicolumn{14}{|c|}{ Sunflower seed meal number ${ }^{1}$} \\
\hline & 1 & 2 & 3 & 4 & 5 & 6 & 7 & 8 & 9 & 10 & Min & Max & Mean & $\mathrm{CV}$ \\
\hline $\mathrm{DM}$ & 90.91 & 91.37 & 92.51 & 90.76 & 92.15 & 92.91 & 91.27 & 91.85 & 90.79 & 90.31 & 90.31 & 92.91 & 91.48 & 0.92 \\
\hline \multicolumn{15}{|l|}{ Composition } \\
\hline $\mathrm{CP}$ & 34.91 & 38.00 & 29.33 & 32.02 & 31.68 & 29.47 & 35.19 & 34.63 & 39.09 & 30.87 & 29.33 & 39.09 & 33.52 & 10.13 \\
\hline Ether extract & 2.19 & 2.08 & 5.23 & 0.93 & 1.23 & 3.47 & 2.02 & 0.88 & 1.73 & 11.33 & 0.88 & 11.33 & 3.11 & 101.99 \\
\hline $\mathrm{NDF}$ & 41.65 & 38.15 & 55.40 & 45.93 & 47.32 & 43.97 & 40.96 & 42.67 & 40.72 & 51.90 & 38.15 & 55.40 & 44.87 & 12.01 \\
\hline $\mathrm{ADF}$ & 25.85 & 24.59 & 37.34 & 31.31 & 31.42 & 28.04 & 25.89 & 27.99 & 26.07 & 30.52 & 24.59 & 37.34 & 28.90 & 13.28 \\
\hline Crude fibre & 21.83 & 23.11 & 36.42 & 30.54 & 29.87 & 27.25 & 21.46 & 25.66 & 22.47 & 33.69 & 21.46 & 36.42 & 27.23 & 19.31 \\
\hline Ash & 6.86 & 7.15 & 5.45 & 6.32 & 6.82 & 7.75 & 8.30 & 6.40 & 6.76 & 6.71 & 5.45 & 8.30 & 6.85 & 11.37 \\
\hline Calcium & 0.27 & 0.32 & 0.17 & 0.34 & 0.33 & 0.45 & 0.44 & 0.30 & 0.30 & 0.31 & 0.17 & 0.45 & 0.32 & 25.07 \\
\hline Phosphorus & 1.04 & 1.15 & 0.63 & 0.81 & 0.94 & 0.84 & 1.02 & 0.98 & 1.06 & 0.92 & 0.63 & 1.15 & 0.94 & 15.89 \\
\hline
\end{tabular}

DM, dry matter; Min, minimum; Max, maximum; CV, coefficient of variation; CP, crude protein; NDF, neutral detergent fibre; ADF, acid detergent fibre.

${ }^{1}$ Sources of sunflower seed meal: 1, 4, and 8 were from Xinjiang; 2, 7, and 9 were from Hebei; 3 was from Liaoning; 5 was from Shanxi; 6 and 10 were from Inner Mongolia. 
Table 2. Analyzed AA composition of sunflower seed meal (\% DM)

\begin{tabular}{|c|c|c|c|c|c|c|c|c|c|c|c|c|c|c|}
\hline \multirow{2}{*}{ Item } & \multicolumn{14}{|c|}{ Sunflower seed meal number ${ }^{1}$} \\
\hline & 1 & 2 & 3 & 4 & 5 & 6 & 7 & 8 & 9 & 10 & $\operatorname{Min}^{2}$ & $\operatorname{Max}^{3}$ & Mean & $\mathrm{CV}^{4}$ \\
\hline \multicolumn{15}{|l|}{ Indispensable AA } \\
\hline Arginine & 2.70 & 3.03 & 2.22 & 2.52 & 2.48 & 2.35 & 2.91 & 2.60 & 3.06 & 2.34 & 2.22 & 3.06 & 2.62 & 11.36 \\
\hline Histidine & 0.89 & 1.11 & 0.87 & 0.86 & 0.85 & 0.80 & 0.97 & 0.88 & 1.18 & 0.83 & 0.80 & 1.18 & 0.93 & 13.67 \\
\hline Isoleucine & 1.36 & 1.55 & 1.20 & 1.29 & 1.25 & 1.15 & 1.45 & 1.34 & 1.62 & 1.23 & 1.15 & 1.62 & 1.34 & 11.45 \\
\hline Leucine & 2.18 & 2.38 & 1.78 & 2.04 & 1.99 & 1.92 & 2.29 & 2.07 & 2.46 & 1.81 & 1.78 & 2.46 & 2.09 & 11.06 \\
\hline Lysine & 1.56 & 1.63 & 1.22 & 1.52 & 1.45 & 1.31 & 1.56 & 1.51 & 1.67 & 1.36 & 1.22 & 1.67 & 1.48 & 9.61 \\
\hline Methionine & 0.80 & 0.86 & 0.61 & 0.74 & 0.76 & 0.65 & 0.80 & 0.77 & 0.89 & 0.66 & 0.61 & 0.89 & 0.75 & 12.00 \\
\hline Phenylalanine & 1.45 & 1.67 & 1.22 & 1.23 & 1.30 & 1.17 & 1.45 & 1.42 & 1.71 & 1.28 & 1.17 & 1.71 & 1.39 & 13.39 \\
\hline Threonine & 1.33 & 1.42 & 1.09 & 1.15 & 1.14 & 1.08 & 1.35 & 1.29 & 1.45 & 1.17 & 1.08 & 1.45 & 1.25 & 11.04 \\
\hline Tryptophan & 0.37 & 0.43 & 0.33 & 0.34 & 0.35 & 0.32 & 0.40 & 0.34 & 0.44 & 0.35 & 0.32 & 0.44 & 0.37 & 11.64 \\
\hline Valine & 1.85 & 2.02 & 1.57 & 1.60 & 1.62 & 1.52 & 1.83 & 1.76 & 2.05 & 1.80 & 1.52 & 2.05 & 1.76 & 10.47 \\
\hline \multicolumn{15}{|l|}{ Dispensable AA } \\
\hline Alanine & 1.70 & 1.76 & 1.41 & 1.55 & 1.54 & 1.49 & 1.74 & 1.66 & 1.79 & 1.52 & 1.41 & 1.79 & 1.62 & 8.10 \\
\hline Aspartate & 3.08 & 3.50 & 2.66 & 2.88 & 2.68 & 2.71 & 3.23 & 3.03 & 3.56 & 2.77 & 2.66 & 3.56 & 3.01 & 11.04 \\
\hline Cystine & 0.64 & 0.62 & 0.39 & 0.52 & 0.53 & 0.47 & 0.60 & 0.60 & 0.71 & 0.48 & 0.39 & 0.71 & 0.56 & 17.15 \\
\hline Glutamine & 7.02 & 7.62 & 5.97 & 6.56 & 6.28 & 6.20 & 7.51 & 6.89 & 7.86 & 6.12 & 5.97 & 7.86 & 6.80 & 10.05 \\
\hline Glycine & 2.05 & 2.32 & 1.70 & 1.93 & 1.91 & 1.69 & 2.12 & 2.01 & 2.35 & 1.92 & 1.69 & 2.35 & 2.00 & 11.22 \\
\hline Proline & 1.19 & 1.30 & 1.12 & 1.16 & 1.17 & 1.11 & 1.28 & 1.17 & 1.33 & 1.16 & 1.11 & 1.33 & 1.20 & 6.58 \\
\hline Serine & 1.45 & 1.64 & 1.14 & 1.38 & 1.37 & 1.29 & 1.53 & 1.41 & 1.66 & 1.36 & 1.14 & 1.66 & 1.42 & 11.00 \\
\hline Tyrosine & 0.78 & 0.93 & 0.66 & 0.68 & 0.63 & 0.70 & 0.88 & 0.75 & 0.97 & 0.59 & 0.59 & 0.97 & 0.76 & 17.58 \\
\hline
\end{tabular}

DM, dry matter; Min, minimum; Max, maximum; CV, coefficient of variation; AA, amino acid.

${ }^{1}$ Sources of sunflower seed meal: 1, 4, and 8 were from Xinjiang; 2, 7, and 9 were from Hebei; 3 was from Liaoning; 5 was from Shanxi; 6 and 10 were from Inner Mongolia.

\section{Chemical analyses}

All analyses in the experiment were performed in duplicate and the chemical analyses were repeated if the difference between duplicates were over $5 \%$. The methods

Table 3. Ingredient composition of experimental diets (\% as-fed)

\begin{tabular}{|c|c|c|}
\hline Ingredients & $\begin{array}{c}\text { Sunflower } \\
\text { seed meal } \\
\text { diets }\end{array}$ & $\begin{array}{c}\text { Nitrogen-free } \\
\text { diet }\end{array}$ \\
\hline Sunflower seed meal & 50.00 & - \\
\hline Corn starch & 35.00 & 73.35 \\
\hline Soybean oil & 2.00 & 3.00 \\
\hline Sucrose & 10.00 & 15.00 \\
\hline Cellulose acetate $^{1}$ & - & 4.00 \\
\hline Limestone & 0.40 & 0.50 \\
\hline Dicalcium phosphate & 1.50 & 2.50 \\
\hline Sodium chloride & 0.30 & 0.45 \\
\hline Chromic oxide & 0.30 & 0.30 \\
\hline Potassium carbonate & - & 0.30 \\
\hline Magnesium oxide & - & 0.10 \\
\hline Micromineral and vitamin premix ${ }^{2}$ & 0.50 & 0.50 \\
\hline \multicolumn{3}{|c|}{$\begin{array}{l}{ }^{1} \text { Made by Chemical Reagents Company, Beijing, China. } \\
{ }^{2} \text { Premix provided the following per kg of complete diet: vitamin A, 5,512 } \\
\mathrm{IU} \text {; vitamin } \mathrm{D}_{3}, 2,200 \mathrm{IU} \text {; vitamin E, } 30 \mathrm{IU} \text {; vitamin } \mathrm{K}_{3}, 2.2 \mathrm{mg} \text {; vitamin } \\
\mathrm{B}_{12}, 27.6 \mu \mathrm{g} \text {; riboflavin, } 4 \mathrm{mg} \text {; pantothenic acid, } 13.8 \mathrm{mg} \text {; niacin, } 30 \mathrm{mg} \text {; } \\
\text { choline chloride, } 400 \mathrm{mg} \text {; folacin, } 0.7 \mathrm{mg} \text {; vitamin } \mathrm{B}_{1}, 1.5 \mathrm{mg} \text {; vitamin } \\
\mathrm{B}_{6}, 3 \mathrm{mg} \text {; biotin, } 44 \mu \mathrm{g} \text {; } \mathrm{Mn}, 40 \mathrm{mg}(\mathrm{MnO}) ; \mathrm{Fe}, 75 \mathrm{mg}\left(\mathrm{FeSO}_{4} \cdot \mathrm{H}_{2} \mathrm{O}\right) ; \mathrm{Zn} \text {, } \\
75 \mathrm{mg}(\mathrm{ZnO}) ; \mathrm{Cu}, 10 \mathrm{mg}\left(\mathrm{CuSO}_{4} \cdot 5 \mathrm{H}_{2} \mathrm{O}\right) ; \mathrm{I}, 0.3 \mathrm{mg}(\mathrm{KI}) ; \mathrm{Se}, 0.3 \mathrm{mg} \\
\left(\mathrm{Na}_{2} \mathrm{SeO}_{3}\right) \text {. }\end{array}$} \\
\hline
\end{tabular}

used to analyze the chemical composition were similar to the description by Ji et al. (2012).

The ten SFSM, diets and digesta samples were analyzed for dry matter (DM) (AOAC procedure 4.1.06, 2000), CP (AOAC procedure 990.03, 2000), Kjeldahl N (Thiex et al., 2002) and CP was calculated as $\mathrm{N} \times 6.25$. The AA were analyzed after being hydrolysed with $6 \mathrm{~N} \mathrm{HCl}$ for $24 \mathrm{~h}$ at $110^{\circ} \mathrm{C}$. Fifteen AA were analyzed using an AA Analyzer (Hitachi L-8900, Tokyo, Japan). Tryptophan was determined after $\mathrm{LiOH}$ hydrolysis for $22 \mathrm{~h}$ at $110^{\circ} \mathrm{C}$ using High Performance Liquid Chromatography (Agilent 1200 series, Santa Clara, CA, USA). Methionine and cystine were determined as methionine sulfone and cysteic acid after cold performic acid oxidation overnight and hydrolysing with $7.5 \mathrm{~N} \mathrm{HCl}$ for $24 \mathrm{~h}$ at $110^{\circ} \mathrm{C}$ using an AA Analyzer (Hitachi L-8800, Tokyo, Japan).

The 10 SFSM were also analyzed for crude fiber (CF), ether extract (EE) (Thiex et al., 2003), ash, calcium (Ca) (AOAC procedure $4.8 .03,2000$ ) and total phosphorus (AOAC procedure 3.4.11, 2000). Neutral detergent fibre (NDF) and acid detergent fibre (ADF) were determined using fibre bags and an analyser (Fibre Analyzer, Ankom Technology, Macedon, NY, USA) following an adaptation procedure described by Van Soest et al. (1991). Diets and ileal digesta samples were analyzed for chromium concentration. A Polarized Zeeman Atomic Absorption Spectrometer (Hitachi Z2000, Tokyo, Japan) was used after 
Table 4. Analyzed composition of experiment diets (\% DM)

\begin{tabular}{|c|c|c|c|c|c|c|c|c|c|c|}
\hline \multirow{2}{*}{ Item } & \multicolumn{10}{|c|}{ Sunflower seed meal number } \\
\hline & 1 & 2 & 3 & 4 & 5 & 6 & 7 & 8 & 9 & 10 \\
\hline$\overline{\mathrm{DM}}$ & 85.67 & 84.16 & 85.87 & 85.19 & 83.49 & 85.92 & 83.98 & 84.13 & 83.54 & 84.76 \\
\hline $\mathrm{CP}$ & 18.94 & 22.77 & 16.09 & 17.84 & 17.49 & 16.80 & 20.38 & 19.20 & 23.06 & 17.16 \\
\hline \multicolumn{11}{|l|}{ Indispensable AA } \\
\hline Arginine & 1.41 & 1.64 & 1.18 & 1.32 & 1.37 & 1.27 & 1.56 & 1.38 & 1.66 & 1.31 \\
\hline Histidine & 0.55 & 0.61 & 0.45 & 0.47 & 0.45 & 0.43 & 0.58 & 0.51 & 0.67 & 0.46 \\
\hline Leucine & 0.73 & 0.90 & 0.65 & 0.66 & 0.68 & 0.63 & 0.81 & 0.69 & 0.92 & 0.65 \\
\hline Isoleucine & 1.06 & 1.24 & 0.96 & 1.04 & 1.12 & 1.05 & 1.20 & 1.00 & 1.33 & 0.97 \\
\hline Lysine & 0.80 & 0.86 & 0.66 & 0.80 & 0.81 & 0.70 & 0.79 & 0.79 & 0.89 & 0.72 \\
\hline Methionine & 0.41 & 0.43 & 0.31 & 0.38 & 0.39 & 0.35 & 0.41 & 0.39 & 0.48 & 0.36 \\
\hline Phenylalanine & 0.77 & 0.95 & 0.65 & 0.70 & 0.83 & 0.66 & 0.80 & 0.76 & 0.97 & 0.70 \\
\hline Threonine & 0.74 & 0.78 & 0.58 & 0.61 & 0.63 & 0.58 & 0.75 & 0.70 & 0.80 & 0.64 \\
\hline Tryptophan & 0.19 & 0.24 & 0.18 & 0.18 & 0.19 & 0.17 & 0.23 & 0.18 & 0.25 & 0.19 \\
\hline Valine & 0.99 & 1.10 & 0.86 & 0.84 & 0.88 & 0.84 & 1.02 & 1.03 & 1.09 & 0.97 \\
\hline \multicolumn{11}{|l|}{ Dispensable AA } \\
\hline Alanine & 0.82 & 0.96 & 0.76 & 0.80 & 0.88 & 0.79 & 0.94 & 0.74 & 0.96 & 0.81 \\
\hline Aspartate & 1.61 & 2.14 & 1.41 & 1.53 & 1.50 & 1.44 & 1.89 & 1.61 & 2.21 & 1.50 \\
\hline Cystine & 0.32 & 0.35 & 0.21 & 0.29 & 0.29 & 0.25 & 0.33 & 0.30 & 0.36 & 0.24 \\
\hline Glutamine & 3.60 & 4.15 & 3.22 & 3.36 & 3.47 & 3.29 & 4.14 & 3.55 & 4.30 & 3.23 \\
\hline Glycine & 1.05 & 1.29 & 0.90 & 1.00 & 1.07 & 0.92 & 1.24 & 1.01 & 1.32 & 1.01 \\
\hline Proline & 0.64 & 0.61 & 0.60 & 0.62 & 0.68 & 0.59 & 0.61 & 0.60 & 0.67 & 0.54 \\
\hline Serine & 0.77 & 0.91 & 0.62 & 0.70 & 0.77 & 0.72 & 0.81 & 0.76 & 0.93 & 0.67 \\
\hline Tyrosine & 0.41 & 0.49 & 0.38 & 0.37 & 0.33 & 0.33 & 0.47 & 0.39 & 0.52 & 0.33 \\
\hline
\end{tabular}

DM, dry matter; $\mathrm{CP}$, crude protein; AA, amino acid.

the samples were prepared by nitric acid-perchloric acid.

\section{Calculations}

Values for AID and SID of CP and each AA were determined according to the method of Stein et al. (2007) described previously.

\section{Statistical analyses}

Simple and multiple regression analyses (stepwise regression analysis) were conducted to determine the relationships among chemical components and AA digestibility. For prediction equations, the residual standard deviation (RSD) was used as the selection criterion. A smaller RSD was proposed to indicate a better fit. Data for AID and SID were analyzed using the Proc-Mixed procedure of SAS (SAS Institute; Cary, NC, USA). Means were calculated using the LSMEANS statement, and when a significant F-test for treatment was observed, means were separated using the PDIFF option. The value of $5 \%$ $(\mathrm{p}<0.05)$ was used to determine significance.

\section{RESULTS}

\section{Composition of ingredients}

The chemical composition for 10 SFSM are shown in Table 1. Among all the ingredients, the CP, EE, NDF, ADF,
$\mathrm{CF}$, Ash, $\mathrm{Ca}$ and $\mathrm{P}$ obviously differed with a coefficient of variation $(\mathrm{CV})$ higher than $10 \%$. The concentration of $\mathrm{EE}$, $\mathrm{CF}, \mathrm{Ca}$ and $\mathrm{P}$ showed the greatest difference with a $\mathrm{CV}$ higher than $15 \%$. On DM basis, the content of CP, NDF, $\mathrm{ADF}$ and $\mathrm{CF}$ ranged from $29.33 \%$ to $39.09 \%, 38.15 \%$ to $55.40 \%, 24.59 \%$ to $37.34 \%$ and $21.46 \%$ to $36.42 \%$, respectively.

The AA composition for SFSM is shown in Table 2. The results showed that most AA were variable $(\mathrm{CV}>10 \%)$ except proline, alanine, and Lys $(\mathrm{CV}<10 \%)$. The average concentration of AA in SFSM were lower than those published by NRC (2012). The level of the four commonly limiting AA were $1.48 \%, 0.75 \%, 1.25 \%$, and $0.37 \%$ for Lys, Met, threonine (Thr) and tryptophan (Trp), respectively.

\section{Ileal digestibility of crude protein and amino acid}

The results of AID and SID are shown in Table 5 and 6 . The AID for CP varied from $60.13 \%$ to $74.72 \%$ among the 10 sources of SFSM. Sunflower seed meal with a high concentration of CP (sources 2 and 9) had higher AID values than SFSM with a lower concentration of $\mathrm{CP}$ (sources 3, 6, and 10) $(\mathrm{p}<0.05)$. The SFSM with the highest concentration of $\mathrm{CP}$ (source 9) had greater digestibility coefficients than the other SFSM for most AA $(p<0.05)$. The results of SID for CP among 10 SFSM were similar to the values for AID. Sources 2 and 9 SFSM had relatively 
Table 5. Apparent ileal digestibility (\%) of CP and AA in sunflower seed meal fed to growing pigs

\begin{tabular}{|c|c|c|c|c|c|c|c|c|c|c|c|c|c|}
\hline \multirow{2}{*}{ Item } & \multicolumn{10}{|c|}{ Sunflower seed meal number } & \multirow{2}{*}{ Mean $^{1}$} & \multirow{2}{*}{ SEM } & \multirow{2}{*}{ p-value } \\
\hline & 1 & 2 & 3 & 4 & 5 & 6 & 7 & 8 & 9 & 10 & & & \\
\hline$\overline{\mathrm{CP}}$ & $67.13^{\mathrm{bcd}}$ & $73.38^{\mathrm{a}}$ & $60.13^{\mathrm{f}}$ & $64.69^{\text {cde }}$ & $66.91^{\text {bcd }}$ & $61.00^{\mathrm{ef}}$ & $69.31^{\mathrm{b}}$ & $68.66^{\mathrm{bc}}$ & $74.72^{\mathrm{a}}$ & $63.09^{\mathrm{def}}$ & 66.90 & 1.35 & $<0.01$ \\
\hline \multicolumn{14}{|l|}{ Indispensable AA } \\
\hline Arginine & $89.19^{\mathrm{a}}$ & $88.19^{\mathrm{a}}$ & $84.93^{\mathrm{bc}}$ & $85.39^{\mathrm{bc}}$ & $87.37^{\mathrm{ab}}$ & $84.40^{\mathrm{c}}$ & $86.68^{\mathrm{abc}}$ & $84.98^{\mathrm{bc}}$ & $88.60^{\mathrm{a}}$ & $86.41^{\mathrm{abc}}$ & 86.61 & 0.88 & $<0.01$ \\
\hline Histidine & $79.25^{\mathrm{abc}}$ & $80.83^{\mathrm{ab}}$ & $67.47^{\mathrm{e}}$ & $75.26^{\mathrm{bcd}}$ & $77.95^{\text {bcd }}$ & $73.56^{\mathrm{d}}$ & $79.82^{\mathrm{abc}}$ & $78.56^{\mathrm{abcd}}$ & $83.65^{\mathrm{a}}$ & $75.00^{\mathrm{cd}}$ & 77.14 & 1.77 & $<0.01$ \\
\hline Leucine & $68.16^{\mathrm{b}}$ & $75.21^{\mathrm{a}}$ & $63.42^{\mathrm{c}}$ & $69.42^{\mathrm{b}}$ & $74.77^{\mathrm{a}}$ & $69.53^{\mathrm{b}}$ & $75.35^{\mathrm{a}}$ & $67.47^{\mathrm{bc}}$ & $74.95^{\mathrm{a}}$ & $66.61^{\mathrm{bc}}$ & 70.49 & 1.53 & $<0.01$ \\
\hline Isoleucine & $73.02^{c}$ & $80.11^{\mathrm{a}}$ & $70.38^{\mathrm{c}}$ & $72.02^{c}$ & $73.89^{\mathrm{bc}}$ & $71.13^{\mathrm{c}}$ & $73.95^{\mathrm{bc}}$ & $68.92^{c}$ & $78.66^{\mathrm{ab}}$ & $68.47^{\mathrm{c}}$ & 73.06 & 1.82 & $<0.01$ \\
\hline Lysine & $76.45^{\mathrm{a}}$ & $78.49^{\mathrm{a}}$ & $63.16^{\mathrm{c}}$ & $75.97^{\mathrm{a}}$ & $75.79^{a}$ & $67.35^{\mathrm{b}}$ & $76.15^{\mathrm{a}}$ & $76.80^{\mathrm{a}}$ & $79.21^{\mathrm{a}}$ & $65.49^{\mathrm{bc}}$ & 73.49 & 1.09 & $<0.01$ \\
\hline Methionine & $84.24^{\mathrm{bc}}$ & $87.78^{\mathrm{ab}}$ & $74.79^{\mathrm{d}}$ & $83.41^{\mathrm{c}}$ & $84.22^{\mathrm{bc}}$ & $80.71^{\mathrm{c}}$ & $84.32^{\mathrm{bc}}$ & $83.11^{\mathrm{c}}$ & $88.60^{\mathrm{a}}$ & $74.92^{d}$ & 82.61 & 1.19 & $<0.01$ \\
\hline Phenylalanine & $72.25^{\mathrm{bc}}$ & $81.99^{\mathrm{a}}$ & $69.80^{\mathrm{c}}$ & $78.01^{\mathrm{ab}}$ & $80.54^{\mathrm{a}}$ & $70.34^{\mathrm{c}}$ & $78.79^{\mathrm{ab}}$ & $78.04^{\mathrm{ab}}$ & $79.39^{\mathrm{a}}$ & $74.98^{\mathrm{abc}}$ & 76.41 & 2.21 & $<0.01$ \\
\hline Threonine & $67.90^{\mathrm{abc}}$ & $70.93^{\mathrm{a}}$ & $55.58^{\mathrm{d}}$ & $62.63^{\mathrm{bc}}$ & $66.72^{\mathrm{abc}}$ & $55.19^{\mathrm{d}}$ & $70.47^{\mathrm{a}}$ & $69.47^{\mathrm{ab}}$ & $72.04^{\mathrm{a}}$ & $61.74^{\mathrm{cd}}$ & 65.27 & 2.30 & $<0.01$ \\
\hline Tryptophan & $69.53^{\mathrm{bc}}$ & $75.68^{\mathrm{a}}$ & $62.53^{\mathrm{d}}$ & $68.95^{\mathrm{bc}}$ & $69.22^{\mathrm{bc}}$ & $64.64^{\mathrm{cd}}$ & $73.56^{\mathrm{ab}}$ & $68.20^{c}$ & $77.40^{\mathrm{a}}$ & $65.42^{\mathrm{cd}}$ & 69.51 & 1.69 & $<0.01$ \\
\hline Valine & $62.91^{\mathrm{de}}$ & $75.39^{\mathrm{a}}$ & $62.00^{\mathrm{e}}$ & $66.79^{\text {cde }}$ & $69.04^{\mathrm{abcd}}$ & $65.56^{\mathrm{de}}$ & $72.89^{\mathrm{abc}}$ & $73.98^{\mathrm{ab}}$ & $75.54^{\mathrm{a}}$ & $68.32^{\text {bcde }}$ & 69.24 & 2.08 & $<0.01$ \\
\hline \multicolumn{14}{|l|}{ Dispensable AA } \\
\hline Alanine & $68.21^{\mathrm{bcd}}$ & $73.56^{\mathrm{a}}$ & $61.55^{\mathrm{ef}}$ & $65.22^{\mathrm{de}}$ & $70.20^{\mathrm{abc}}$ & $66.19^{\mathrm{cd}}$ & $72.06^{\mathrm{ab}}$ & $64.03^{\mathrm{de}}$ & $74.02^{\mathrm{a}}$ & $59.64^{\mathrm{f}}$ & 67.47 & 1.43 & $<0.01$ \\
\hline Aspartate & $69.61^{\mathrm{bc}}$ & $79.59^{\mathrm{a}}$ & $59.63^{\mathrm{d}}$ & $70.36^{\mathrm{bc}}$ & $71.37^{\mathrm{b}}$ & $66.85^{\mathrm{c}}$ & $75.71^{\mathrm{a}}$ & $71.36^{\mathrm{b}}$ & $76.66^{\mathrm{a}}$ & $69.59^{\mathrm{bc}}$ & 71.07 & 1.34 & $<0.01$ \\
\hline Cystine & $62.76^{\mathrm{de}}$ & $71.26^{\mathrm{ab}}$ & $39.76^{\mathrm{g}}$ & $63.82^{\mathrm{cd}}$ & $67.27^{\mathrm{bcd}}$ & $57.80^{\mathrm{ef}}$ & $68.65^{\mathrm{abc}}$ & $64.20^{\mathrm{cd}}$ & $74.06^{\mathrm{a}}$ & $55.70^{\mathrm{f}}$ & 62.53 & 1.83 & $<0.01$ \\
\hline Glutamine & $79.28^{\mathrm{b}}$ & $84.04^{\mathrm{a}}$ & $75.13^{\mathrm{c}}$ & $79.71^{\mathrm{b}}$ & $80.94^{\mathrm{b}}$ & $79.34^{\mathrm{b}}$ & $83.84^{\mathrm{a}}$ & $80.38^{\mathrm{b}}$ & $83.78^{\mathrm{a}}$ & $79.32^{\mathrm{b}}$ & 80.58 & 0.80 & $<0.01$ \\
\hline Glycine & $55.89^{d}$ & $60.93^{c}$ & $44.94^{\mathrm{e}}$ & $47.17^{\mathrm{e}}$ & $55.34^{\mathrm{d}}$ & $48.60^{\mathrm{e}}$ & $66.19^{\mathrm{b}}$ & $58.20^{\mathrm{cd}}$ & $70.02^{\mathrm{a}}$ & $47.88^{\mathrm{e}}$ & 55.52 & 1.34 & $<0.01$ \\
\hline Proline & $71.86^{\mathrm{b}}$ & $71.58^{\mathrm{b}}$ & $42.80^{\mathrm{f}}$ & $67.28^{\mathrm{c}}$ & $51.50^{\mathrm{e}}$ & $61.20^{\mathrm{d}}$ & $70.22^{\mathrm{bc}}$ & $67.43^{\mathrm{c}}$ & $77.54^{\mathrm{a}}$ & $62.28^{\mathrm{d}}$ & 64.37 & 1.03 & $<0.01$ \\
\hline Serine & $67.00^{\mathrm{bc}}$ & $74.50^{\mathrm{a}}$ & $49.40^{\mathrm{e}}$ & $67.50^{\mathrm{cd}}$ & $63.53^{\mathrm{bc}}$ & $61.66^{\mathrm{d}}$ & $69.19^{\mathrm{b}}$ & $69.73^{\mathrm{b}}$ & $75.36^{\mathrm{a}}$ & $60.14^{\mathrm{d}}$ & 65.78 & 1.59 & $<0.01$ \\
\hline Tyrosine & $83.93^{\mathrm{b}}$ & $86.34^{\mathrm{a}}$ & $76.35^{\mathrm{e}}$ & $78.59^{\mathrm{d}}$ & $78.07^{\mathrm{de}}$ & $67.19^{\mathrm{f}}$ & $84.46^{\mathrm{ab}}$ & $80.54^{\mathrm{c}}$ & $84.90^{\mathrm{ab}}$ & $64.38^{\mathrm{g}}$ & 78.48 & 0.67 & $<0.01$ \\
\hline
\end{tabular}

CP, crude protein; AA, amino acid; SEM, standard error of the mean.

${ }^{1}$ The average value of 10 sunflower seed meal.

${ }^{\mathrm{a}-\mathrm{g}}$ Values within the same row with no common superscript differ significantly $(\mathrm{p}<0.05)$.

higher SID for CP than the other sources $(\mathrm{p}<0.05)$. However, concentration of CP (source 1$)(\mathrm{p}<0.05)$ which was a bit the SID value of one sample with a lower concentration of different compared with the result of AID.

CP (source 5) was higher than another sample with a higher Among indispensable AA of SFSM, the AID of Lys,

Table 6. Standardized ilea digestibility (\%) of CP and AA in sunflower seed meal fed to growing pigs

\begin{tabular}{|c|c|c|c|c|c|c|c|c|c|c|c|c|c|}
\hline \multirow{2}{*}{ Item } & \multicolumn{10}{|c|}{ Sunflower seed meal number } & \multirow{2}{*}{ Mean $^{1}$} & \multirow{2}{*}{ SEM } & \multirow{2}{*}{ p-value } \\
\hline & 1 & 2 & 3 & 4 & 5 & 6 & 7 & 8 & 9 & 10 & & & \\
\hline$\overline{\mathrm{CP}}$ & $72.71^{\mathrm{cd}}$ & $78.03^{\mathrm{ab}}$ & $66.70^{\mathrm{e}}$ & $70.62^{\text {cde }}$ & $72.95^{\text {cd }}$ & $67.30^{\mathrm{e}}$ & $74.49^{\mathrm{bc}}$ & $74.16^{\mathrm{bc}}$ & $79.31^{\mathrm{a}}$ & $69.25^{\mathrm{de}}$ & 72.55 & 1.35 & 0.01 \\
\hline \multicolumn{14}{|l|}{ Indispensable AA } \\
\hline Arginine & $91.75^{\mathrm{a}}$ & $90.39^{\mathrm{abc}}$ & $88.00^{\mathrm{bcd}}$ & $88.11^{\mathrm{bcd}}$ & $90.02^{\text {abcd }}$ & $87.25^{\mathrm{d}}$ & $88.99^{\text {abcd }}$ & $87.60^{\text {cd }}$ & $90.77^{\mathrm{ab}}$ & $89.18^{\text {abcd }}$ & 89.21 & 0.88 & $<0.01$ \\
\hline Histidine & $81.83^{\mathrm{abc}}$ & $83.16^{\mathrm{ab}}$ & $70.66^{d}$ & $78.31^{\mathrm{bc}}$ & $81.10^{\mathrm{abc}}$ & $76.84^{\mathrm{c}}$ & $82.29^{\mathrm{abc}}$ & $81.36^{\mathrm{abc}}$ & $85.79^{\mathrm{a}}$ & $78.11^{\mathrm{bc}}$ & 79.94 & 1.77 & $<0.01$ \\
\hline Leuci & $72.01^{\mathrm{bc}}$ & $78.51^{\mathrm{a}}$ & $67.66^{\mathrm{c}}$ & $73.37^{\mathrm{b}}$ & $78.41^{\mathrm{a}}$ & $73.44^{\mathrm{b}}$ & $78.75^{\mathrm{a}}$ & $71.57^{\mathrm{bc}}$ & $78.03^{\mathrm{a}}$ & $70.83^{\mathrm{bc}}$ & 74.26 & 1.53 & $<0.01$ \\
\hline Isoleuc & $75.81^{\mathrm{bc}}$ & $82.37^{\mathrm{a}}$ & $73.47^{\mathrm{c}}$ & $75.11^{\mathrm{c}}$ & $76.88^{\mathrm{bc}}$ & $74.35^{\mathrm{c}}$ & $76.46^{\mathrm{bc}}$ & $71.85^{\mathrm{c}}$ & $80.87^{\mathrm{ab}}$ & $71.58^{\mathrm{c}}$ & 75.87 & 1.82 & 0.01 \\
\hline Lysine & $79.63^{\mathrm{a}}$ & $81.46^{\mathrm{a}}$ & $67.03^{c}$ & $79.17^{\mathrm{a}}$ & $78.96^{\mathrm{a}}$ & $70.99^{\mathrm{b}}$ & $79.40^{\mathrm{a}}$ & $80.04^{\mathrm{a}}$ & $82.07^{\mathrm{a}}$ & $69.02^{\mathrm{bc}}$ & 76.78 & 1.09 & $<0.01$ \\
\hline Methionine & $86.17^{\mathrm{bc}}$ & $89.63^{\mathrm{ab}}$ & $77.39^{d}$ & $85.54^{\mathrm{c}}$ & $86.26^{\mathrm{bc}}$ & $82.99^{c}$ & $86.25^{\mathrm{bc}}$ & $85.14^{\mathrm{c}}$ & $90.27^{\mathrm{a}}$ & $77.16^{\mathrm{d}}$ & 84.68 & 1.19 & $<0.01$ \\
\hline Phen & $74.46^{\mathrm{bc}}$ & $83.78^{\mathrm{a}}$ & $72.42^{\mathrm{c}}$ & $80.45^{\mathrm{ab}}$ & $82.58^{\mathrm{a}}$ & $72.93^{\mathrm{c}}$ & $80.90^{\mathrm{ab}}$ & $80.27^{\mathrm{ab}}$ & $81.15^{\mathrm{ab}}$ & $77.41^{\mathrm{abc}}$ & 78.64 & 2.21 & $<0.01$ \\
\hline Threo & $73.29^{\mathrm{abc}}$ & $76.04^{\mathrm{ab}}$ & $62.39^{\mathrm{de}}$ & $69.18^{\text {bcd }}$ & $72.97^{\mathrm{abc}}$ & $61.97^{\mathrm{e}}$ & $75.75^{\mathrm{ab}}$ & $75.10^{\mathrm{abc}}$ & $77.01^{\mathrm{a}}$ & $67.93^{\text {cde }}$ & 71.16 & 2.30 & $<0.01$ \\
\hline Trypt & $75.41 b^{\text {cd }}$ & $80.15^{\mathrm{ab}}$ & $68.70^{\mathrm{e}}$ & $75.11^{\mathrm{bcd}}$ & $74.92^{\mathrm{bcd}}$ & $71.02^{\mathrm{de}}$ & $78.21^{\mathrm{abc}}$ & $74.41^{\text {cd }}$ & $81.80^{\mathrm{a}}$ & $71.06^{\mathrm{de}}$ & 75.08 & 1.69 & $<0.01$ \\
\hline Valine & $66.69^{d}$ & $78.79^{\mathrm{a}}$ & $66.36^{\mathrm{d}}$ & $71.20^{\text {bcd }}$ & $73.26^{\mathrm{abc}}$ & $69.99^{\text {cd }}$ & $76.56^{\mathrm{ab}}$ & $77.58^{\mathrm{ab}}$ & $78.94^{\mathrm{a}}$ & $72.16^{\mathrm{bcd}}$ & 73.15 & 2.08 & $<0.01$ \\
\hline \multicolumn{14}{|l|}{ Dispensable AA } \\
\hline Alanine & $.41^{\text {bcd }}$ & $78.04^{\mathrm{a}}$ & $67.16^{\mathrm{ef}}$ & $70.55^{\mathrm{de}}$ & $75.06^{\mathrm{abc}}$ & $71.61^{\circ}$ & $76.59^{\mathrm{ab}}$ & $69.78^{\text {de }}$ & $78.48^{\mathrm{a}}$ & $64.90^{\mathrm{f}}$ & 72.56 & 1.43 & $<0.01$ \\
\hline Aspartate & $72.78^{\text {cd }}$ & $81.98^{\mathrm{a}}$ & $63.24^{\mathrm{e}}$ & $73.70^{\text {cd }}$ & $74.77^{\mathrm{bc}}$ & $70.39^{d}$ & $78.42^{\mathrm{ab}}$ & $74.52^{\text {bcd }}$ & $78.97^{\mathrm{a}}$ & $73.00^{\text {cd }}$ & 74.18 & 1.34 & $<0.01$ \\
\hline Cystine & $68.64^{\text {bcd }}$ & $76.64^{\mathrm{a}}$ & $48.56^{\mathrm{e}}$ & $70.24^{\mathrm{bc}}$ & $73.65^{\mathrm{ab}}$ & $65.27^{\mathrm{cd}}$ & $74.30^{\mathrm{ab}}$ & $70.35^{\mathrm{bc}}$ & $79.27^{\mathrm{a}}$ & $63.43^{\mathrm{d}}$ & 69.03 & 1.83 & $<0.01$ \\
\hline Glutan & $81.31^{\mathrm{b}}$ & $85.79^{\mathrm{a}}$ & $77.39^{\mathrm{c}}$ & $81.88^{\mathrm{b}}$ & $83.04^{\mathrm{b}}$ & $81.56^{\mathrm{b}}$ & $85.60^{\mathrm{a}}$ & $82.43^{b}$ & $85.48^{\mathrm{a}}$ & $81.58^{b}$ & 82.61 & 0.80 & $<0.01$ \\
\hline Gly & $63.72^{\mathrm{bc}}$ & $67.32^{\mathrm{b}}$ & $54.15^{\mathrm{d}}$ & $55.43^{\mathrm{d}}$ & $63.07^{\mathrm{c}}$ & $57.54^{\mathrm{d}}$ & $72.82^{\mathrm{a}}$ & $66.39^{\mathrm{bc}}$ & $76.29^{\mathrm{a}}$ & $56.08^{d}$ & 63.28 & 1.34 & $<0.01$ \\
\hline Proline & $79.79^{\mathrm{b}}$ & $79.84^{\mathrm{b}}$ & $51.24^{\mathrm{f}}$ & $75.44^{\mathrm{c}}$ & $58.96^{\mathrm{e}}$ & $69.75^{\mathrm{d}}$ & $78.48^{\mathrm{bc}}$ & $75.94^{\mathrm{c}}$ & $85.06^{\mathrm{a}}$ & $71.73^{\mathrm{d}}$ & 72.62 & 1.03 & $<0.01$ \\
\hline Serine & $72.21^{\mathrm{bc}}$ & $78.94^{\mathrm{a}}$ & $55.91^{\mathrm{e}}$ & $69.08^{\mathrm{cd}}$ & $72.75^{\mathrm{bc}}$ & $67.30^{\mathrm{d}}$ & $74.15^{\mathrm{b}}$ & $75.02^{\mathrm{ab}}$ & $79.70^{\mathrm{a}}$ & $66.14^{\mathrm{d}}$ & 71.12 & 1.59 & $<0.01$ \\
\hline Tyrosine & $93.01^{\mathrm{a}}$ & $93.93^{\mathrm{a}}$ & $86.20^{\mathrm{c}}$ & $88.58^{\mathrm{b}}$ & $89.45^{\mathrm{b}}$ & $78.53^{\mathrm{d}}$ & $92.40^{\mathrm{a}}$ & $90.13^{\mathrm{b}}$ & $92.15^{\mathrm{a}}$ & $75.74^{\mathrm{e}}$ & 88.01 & 0.67 & $<0.01$ \\
\hline
\end{tabular}

CP, crude protein; AA, amino acid; SEM, standard error of the mean.

${ }^{1}$ The average value of 10 sunflower seed meal.

${ }^{\mathrm{a}-\mathrm{f}}$ Values within the same row with no common superscript differ significantly $(\mathrm{p}<0.05)$. 
Met, Thr, and Trp in the sample with the highest concentration of CP (source 9) was the highest at $79.21 \%$, $88.60 \%, 72.04 \%$, and $77.40 \%$, respectively. Also, the SID of most AA in sources 2, 7, and 9 were the highest among all SFSM. Source 2 had relatively higher AID and SID values for leucine and isoleucine than other SFSM ( $\mathrm{p}<0.05)$. The AID of phenylalanine in source 5 was $80.54 \%$ which was beyond the average level $(\mathrm{p}<0.05)$. Additionally, SFSM (sources 3 and 10) with lower concentrations of indispensable AA had lower AID and SID than other SFSM $(\mathrm{p}<0.05)$.

Correlations and prediction equations for nitrogen and amino acid digestibility

Several factors affected the prediction equations for $\mathrm{N}$ and AA. Among the representative chemical constituents (\% of DM), EE had a negative correlation with Lys and Met $(p<0.05)$. The best single predictor for AID and SID was Met according to a linear stepwise regression. The best fit equations were obtained for AID N and SID N, which were: AID $\mathrm{N}=22.97+(0.74 \times \mathrm{CP})+(25.44 \times \mathrm{Met})$ with $\mathrm{R}^{2}=0.96$, $\mathrm{RSD}=0.87, \mathrm{p}<0.05 ;$ and SID N $=35.22+(0.54 \times \mathrm{CP})+(25.38$ $\times$ Met) with $\mathrm{R}^{2}=0.96, \mathrm{RSD}=0.83, \mathrm{p}<0.05$. In addition, $\mathrm{CP}$, Trp and $\mathrm{Ca}$ were also used to estimate the digestibility in different equations (Table 7).

\section{DISCUSSION}

\section{Composition of ingredient}

According to the reports, SFSM is a valuable ingredient for swine because of its high energy concentration and ease of handing in feed mills (Thacker, 1998). It is also a good ingredient because it is free of most antinutritional factors (Wahlstrom, 1990).

The results of the chemical analysis of SFSM conducted in this experiment varied greatly. The $\mathrm{CV}$ of $\mathrm{CP}, \mathrm{NDF}, \mathrm{ADF}$,
$\mathrm{CF}$, Ash, $\mathrm{Ca}$, and $\mathrm{P}$ was higher $(\mathrm{CV}>10 \%)$ due to the selection of samples before the official experiment. Preparations for collecting samples from the major provinces which cultivate SFSM in China involved contacting the representative plants, acquiring basic information on the chemical concentration of SFSM in each plant and collecting typical samples in person. In addition, the variation of SFSM might result from different growth conditions, such as climate and soil conditions (Alpaslan and Gündüz, 2000). Although, most SFSM underwent a similar production process of a pre-pressing extraction method (Fick and Miller, 1997). The differences, such as temperature, pressure and time during the production process might lead to the changes in chemical concentration in SFSM (Clandinin and Robblee, 1950; Parrado et al., 1991). The large variation of $\mathrm{EE}(0.88 \%$ to $11.33 \%)$ was mainly related to the different extraction process.

The average concentration of CP (sources 1, 2, 7, 8, and 9) were higher than other sources and NRC (2012) probably because of genetic improvement (Robertson, 1972). The concentrations of EE among the ten SFSM sources varied from $0.88 \%$ to $11.33 \%$ depending on the extraction process and the amount of residual oil left after extraction (Dinusson, 1990). The EE in source 4 and 8 SFSM were lower than the others which means the oil had been extracted according to the Association of American Feed Control Officials (AAFCO, 2011). The 10 SFSM had an higher average value of NDF but lower ADF than González-Vega et al. (2012) which was similar to the value reported in NRC (2012).

\section{Digestibility of crude protein and amino acid}

Waldroup et al. (1970) reported that SFSM can be effectively used to replace up to $50 \%$ of SBM in broiler diets. The AID and SID values for CP in this experiment were lower than NRC (2012) because of the lower

Table 7. Linear regression equations for prediction of AA digestibility (\%) based on the chemical composition (\% of DM) of sunflower seed meal fed to growing pigs ${ }^{1}$

\begin{tabular}{|c|c|c|c|c|}
\hline Number & Linear regression equations & $\mathrm{RSD}^{2}$ & $\mathrm{R}^{2}$ & p-value \\
\hline 1 & AID N $=22.97+(0.74 \times \mathrm{CP})+(25.44 \times \mathrm{Met})$ & 0.87 & 0.96 & $<0.01$ \\
\hline 2 & AID Lys $=30.00-(0.62 \times E E)+(30.70 \times$ Lys $)$ & 0.78 & 0.97 & $<0.01$ \\
\hline 3 & AID Lys $=34.04+(94.25 \times$ Met $)-(86.16 \times \operatorname{Trp})$ & 0.85 & 0.98 & $<0.01$ \\
\hline 4 & AID Met $=53.56-(0.48 \times \mathrm{EE})+(8.52 \times \mathrm{Ca})+(36.86 \times \mathrm{Met})$ & 0.77 & 0.97 & $<0.01$ \\
\hline 5 & AID $\mathrm{Thr}=17.22+(63.72 \times \mathrm{Met})$ & 2.17 & 0.87 & $<0.01$ \\
\hline 6 & SID N $=35.22+(0.54 \times C P)+(25.38 \times \mathrm{Met})$ & 0.83 & 0.96 & $<0.01$ \\
\hline 7 & SID Lys $=36.24-(0.61 \times E E)+(28.70 \times$ Lys $)$ & 0.93 & 0.97 & $<0.01$ \\
\hline 8 & SID Lys $=39.65+(90.30 \times$ Met $)-(84.36 \times \operatorname{Trp})$ & 0.84 & 0.98 & $<0.01$ \\
\hline 9 & SID Met $=58.02-(0.49 \times \mathrm{EE})+(7.96 \times \mathrm{Ca})+(33.98 \times \mathrm{Met})$ & 0.77 & 0.97 & $<0.01$ \\
\hline 10 & SID Thr $=28.39+(56.73 \times$ Met $)$ & 2.04 & 0.87 & $<0.01$ \\
\hline
\end{tabular}

AA, amino acid; DM, dry matter; RSD, residual standard deviation; AID, apparent ileal digestibility; CP, crude protein; Met, methionine; Lys, lysine; EE, ether extract; Trp, tryptophan; Thr, threonine; SID, standardized ileal digestibility.

${ }^{1}$ Regression equations were developed based on stepwise regression analyses.

${ }^{2} \mathrm{RSD}=$ The root mean square of the error that applies to the whole model. 
concentration of CP in the 10 SFSM. The results of AID and SID of CP and AA in source 3 SFSM resulted from the highest concentration of NDF, ADF, and CF which had depressive effect on AA digestibility (Sauer et al., 1980; Lenis et al., 1996).

The SFSM with relatively higher concentration of EE associated with lower NDF and ADF (source 2) had higher AID and SID values $(p<0.05)$. The different content of EE in the diets also affected the digestibility of ten sunflower seed meal (Noblet and Perez, 1993). The relatively higher concentration of NDF and CF may contribute to a decreased AA digestibility (Sauer et al., 1980; Lenis et al., 1996).

Jørgensen et al. (1984) reported that the digestibility of sunflower seed products is lower than SBM. Sunflower seed meal has a lower content of Lys than SBM (Smith, 1968; Sosulski and Sarwar, 1973). Sources 2 and 9 SFSM had a higher concentration of Lys. However, the average AID and SID values of Lys among all SFSM were lower than NRC (2012). Differences in heating temperature probably also caused the variation of AID and SID value of $\mathrm{CP}$ resulting in Maillard reaction which decreased the concentration and digestibility of Lys in corn distillers dried grains with solubles (Pahm et al., 2008). In addition, the concentration of Met was relatively higher in SFSM than SBM (Olvera-Novoa et al., 2002).

For most indispensable AA, the AID was lower than the value shown in NRC (2012). However, the SID of those AA had less variation. This means that endogenous loss of AA can not be ignored for evaluation of AA. In addition, the SID value had greater accuracy than AID (González-Vega et al., 2012).

\section{Correlations and prediction equation of nitrogen and amino acid digestibility}

The results of the study indicated that the variability of chemical composition in SFSM could contribute to the difference of AID and SID values. The AID and SID of CP could be accurately predicted by Met which means that Met was highly correlated with $\mathrm{CP}$. The concentration of reactive Lys was a good predictor for the concentration of Lys in distillers dried grains with solubles and canola meal, respectively (Almeida et al., 2014). The Lys concentration per unit CP could be an acceptable predictor of Lys SID in wheat distillers dried grains with solubles (Cozannet et al., 2010). Other factors, such as CP, Trp, and Ca were also essential for estimating the digestibility of AA.

In conclusion, the digestibility of SFSM has great variation resulting from differences in chemical composition. The concentration of Met was the major factor affected in the equations established in this experiment. In order to improve SFSM as an alternative to SBM for use in diets for swine, more SFSM samples should be collected and further research should be conducted to increase the accuracy of the equations.

\section{ACKNOWLEDGMENTS}

This research was financially supported by the Special Public Sector Fund in Agriculture (200903006) and National Natural Science Foundation of China (31372316).

\section{REFERENCES}

AAFCO. 2011. Official Publication. Assoc. Am. Feed Control Off. Inc. Washington, DC, USA.

Adeola, O. 2001. Digestion and balance techniques in pigs. In: Swine Nutrition, 2nd ed. (Eds. D. J. Lewis and L. L. Southern). CRC Press, New York, USA. pp. 903-916.

Almeida F. N., J. K. Htoo, J. Thomson, and H. H. Stein. 2014. Effects of heat treatment on the apparent and standardized ileal digestibility of amino acids in canola meal fed to growing pigs. Anim. Feed Sci. Technol. 187:44-52.

Alpaslan, M. and H. Gündüz. 2000. The effects of growing conditions on oil content, fatty acid composition and tocopherol content of some sunflower seed varieties produced in Turkey. Food/Nahrung 44:434-437.

AOAC. 2000. Official Methods of Analysis, 17th ed. Association of Official Analytical Chemists, Arlington, VA, USA.

Baidoo, S. K., B. N. Mitaru, F. X. Aherne, and R. Blair. 1987. The nutritive value of canola meal for early-weaned pigs. Anim. Feed Sci. Technol. 18:45-53.

Batal, A., N. Dale, and M. Café. 2005. Nutrient composition of peanut meal. J. Appl. Poult. Res. 14:254-257.

Brand, T. S., D. A. Brandt, and C. W. Cruywagen. 2001. Utilisation of growing-finishing pig diets containing high levels of solvent or expeller oil extracted canola meal. NZ J. Agric. Res. 44:31-35.

Church, D. C. and R. O. Kellems. 1998. Supplemental protein sources. In: Livestock Feeds and Feeding, 4th ed. (Eds. R. O. Kellems and D. C. Church). Prentice-Hall, Upper Saddle River, NJ, USA. pp. 135-163.

Clandinin, D. R. and A. R. Robblee. 1950. The effects of methods of processing on the nutritive value of sunflower seed meals. Poult. Sci. 29:753.

Cozannet, P., Y. Primot, C. Gady, J. P. Métayer, P. Callu, M. Lessire, F. Skiba, and J. Noblet. 2010. Ileal digestibility of amino acids in wheat distillers dried grains with solubles for pigs. Anim. Feed Sci. Technol. 158:177-186.

Cromwell, G. L. 2000. Utilization of soy products in swine diets. In: Soy in Animal Nutrition (Ed. J. K. Drackley). Fed. Anim. Sci. Soc. Savoy, IL, USA. pp. 258-282.

Dinusson, W. E. 1990. Sunflower seed meal. In: Nontraditional Feed Sources in Swine Production (Eds. P. A. Thacker and R. N. Kirkwood). Butterworths, Stoneham, MA, USA. pp. 465472.

FAO. 2012. Oil, sunflower seed and sunflower seed seed. http://faostat3.fao.org/faostat-gateway/go/to/search/sunflower seed/E. Accessed January 17, 2014.

Fick, G. N. and J. F. Miller. 1997. Sunflower breeding. In: Sunflower Technology and Production (Ed. A. A. Schneiter). ASA-CSSA-SSSA, Madison, WI, USA. pp. 395-439. 
Flagella, Z., T. Rotunno, E. Tarantino, R. Di Caterina, and A. De Caro. 2002. Changes in seed yield and oil fatty acid composition of high oleic sunflower (Helianthus annuus L.) hybrids in relation to the sowing date and the water regime. Eur. J. Agron. 17:221-230.

González-Vega, J. C. and H. H. Stein. 2012. Amino acid digestibility in canola, cottonseed, and sunflower products fed to finishing pigs. J. Anim. Sci. 90:4391-4400.

Ji, Y., L. Zuo, F. L. Wang, D. F. Li, and C. H. Lai. 2012. Nutritional value of 15 corn gluten meals for growing pigs: Chemical composition, energy content and amino acid digestibility. Arch. Anim. Nutr. 66:283-302.

Jørgensen, H., W. C. Sauer, and P. A. Thacker. 1984. Amino acid availabilities in soybean meal, sunflower meal, fish meal and meat and bone meal fed to growing pigs. J. Anim. Sci. 58:926934

Just, A., H. Jorgensen, J. A. Femandez, S. Beth-Andersen, and N. Enggaard Hansen. 1983. The chemical composition, digestibility, energy and protein value of different feedstuffs for pigs. Commun. No. 556 from the National Institute of Animal Science, Denmark, pp. 99 (In Danish with English summary).

Lenis, N. P., P. Bikker, J. van der Meulen, J. Th. M. van Diepen, J. G. M. Bakker, and A. W. Jongbloed. 1996. Effect of dietary neutral detergent fiber on ileal digestibility and portal flux of nitrogen and amino acids and on nitrogen utilization in growing pigs. J. Anim. Sci. 74:2687-2699.

Milic, B., S. Stojanovic, N. Vucurevic, and M. Turcic. 1968. Chlorogenic and quinic acids in sunflower meal. J. Sci. Food Agric. pp. 19:108-113.

Noblet, J. and J. M. Perez. 1993. Prediction of digestibility of nutrients and energy values of pig diets from chemical analysis. J. Anim. Sci. 71:3389-3398.

Noland, P. R., M. Funderburg, J. Atteberry, and K. W. Scott. 1968. Use of glandless cottonseed meal in diets for young pigs. J. Anim. Sci. 27:1319-1321.

NRC. 2012. Nutrient Requirements of Swine. 11th rev ed. National Academy Press, Washington DC, USA.

Olvera-Novoa, M. A., L. Olivera-Castillo, and C. A. MartínezPalacios. 2002. Sunflower meal as a protein source in diets for Tilapia rendalli (Boulanger, 1896) fingerlings. Aquac. Res. 33:223-229.

Opalka, M., L. Dusza, M. Koziorowski, J. Staszkiewicz, K. Lipinski, and J. Tywonczuk. 2001. Effect of long-term feeding with graded levels of low glucosinolate rapeseed meal on endocrine status of gilts and their piglets. Livest. Prod. Sci. 69: 233-243.

Pahm, A. A., C. Pedersen, D. Hoehler, and H. H. Stein. 2008. Factors affecting the variability in ileal amino acid digestibility in corn distillers dried grains with solubles fed to growing pigs. J. Anim. Sci. 86:2180-2189.

Parrado, J., J. Bautista, and A. Machado. 1991. Production of soluble enzymic protein hydrolyzate from industrially defatted nondehulled sunflower meal. J. Agric. Food Chem. 39:447-450.

Robertson, J. A. 1972. Sunflowers: America's neglected crop. J. Am. Oil Chem. Soc. 49:239-244.

Salunkhe, D. K., J. K. Chavan, R. N. Adsule, and S. S. Kadam. 1992. World Oilseeds: Chemistry, Technology and Utilization. Van Nostrand Reinhold, New York, NY, USA. pp. 140-216.
Sasipriya, G. and P. Siddhuraju. 2013. Evaluation of growth performance, serum biochemistry and haematological parameters on broiler birds fed with raw and processed samples of Entada scandens, Canavalia gladiata and Canavalia ensiformis seed meal as an alternative protein source. Trop. Anim. Health Prod. 45:811-820.

Sauer, W. C., A. Just, H. H. Jørgensen, M. Fekadu, and B. O. Eggum. 1980. The influence of diet composition on the apparent digestibility of crude protein and amino acids at the terminal ileum and overall in pigs. Acta Agric. Scand. 30:449459.

Seerley, R. W., D. Burdick, W. C. Russom, R. S. Lowrey, H. C. McCampbell, and H. E. Amos. 1974. Sunflower meal as a replacement for soybean meal in growing swine and rat diets. J. Anim. Sci. 38:947-953.

Senkoylu, N. and N. Dale. 1999. Sunflower meal in poultry diets: A review. World Poult. Sci. J. 55:153-174.

Smith, K. J. 1968. A review of the nutritional value of sunflower meal. Feedstuffs 40:20.

Sosulski, F. W. and G. Sarwar. 1973. Amino acid composition of oilseed meals and protein isolates. Can. Inst. Food Sci. Technol. J. 6:1-5.

Stein, H. H., B. Sève, M. F. Fulle, P. J. Moughan, and C. F. M. de Lange. 2007. Invited review: Amino acid bioavailability and digestibility in pig feed ingredients: Terminology and application. J. Anim. Sci. 85:172-180.

Stein, H. H., C. F. Shipley, and R. A. Easter. 1998. Technical note: A technique for inserting a T-cannula into the distal ileum of pregnant sows. J. Anim. Sci. 76:1433-1436.

Sulabo R. C., W. S. Ju, and H. H. Stein. 2013. Amino acid digestibility and concentration of digestible and metabolizable energy in copra meal, palm kernel expellers and palm kernel meal fed to growing pigs. J. Anim. Sci. 91:1391-1399.

Tanksley, T. D., D. A. Knabe, P. Kenneth, T. Zebrowska, and J. R. Corley. 1981. Apparent digestibility of amino acids and nitrogen in three cottonseed meals and one soybean meals. J. Anim. Sci. 52:769-777.

Thacker, P. A. 1998. Effect of micronization of full-fat canola seed on performance and carcass characteristics of growingfinishing pigs. Anim. Feed Sci. Technol. 71:89-97.

Thiex, N. J., H. Manson, S. Anderson, and J. A. Persson. 2002. Determination of crude protein in animal feed, forage, grain and oilseeds by using block digestion with copper catalyst and steam distillation into boric acid: Collaborative study. J. AOAC Int. 85:309-317.

Thiex N. J., S. Anderson, and B. Gildemeister. 2003. Crude fat, diethyl ester extraction, in feed, cereal grain, and forage (Randall/Soxtec/submersion method): Collaborative study. J. AOAC. Int. 86:888-898.

Van Soest, P. J., J. B. Robertson, and B. A. Lewis. 1991. Methods for dietary fiber and non-starch polysaccharides in relation to animal nutrition. J. Dairy Sci. 74:3568-3597.

Wahlstrom, R. C. 1990. Sunflower seeds. In: Nontraditional Feed Sources in Swine Production (Eds. P. A. Thacker and R. N. Kirkwood). Butterworths, Stoneham, MA, USA. pp. 473-480.

Waldroup, P. W., C. M. Hillard, and R. J. Mitchell. 1970. Sunflower meal as a protein supplement for broiler diets. Feedstuffs 42:41.

Zhang, H. Y., J. Q. Yi, X. S. Piao, P. F. Li, Z. K. Zeng, D. Wang, L. 
Liu, G. Q. Wang, and X. Han. 2013. The metabolizable energy value, standardized ileal digestibility of amino acids in soybean meal, soy protein concentrate and fermented soybean meal and the application of these products in early-weaned piglets. Asian Australas. J. Anim. Sci. 26:691-699.
Zhang, T., L. Liu, and X. S. Piao. 2012. Predicting the digestible energy of rapeseed meal from its chemical composition in growing-finishing pigs. Asian Australas. J. Anim. Sci. 25:375381. 